Proceedings

\title{
Antioxidant Activity of Film Forming Systems Based on Melanins from 5,6-dihydroxyindole Derivatives ${ }^{+}$
}

\author{
Rita Argenziano *, Maria Laura Alfieri, Lucia Panzella, Alessandra Napolitano and Marco \\ d'Ischia \\ Department of Chemical Sciences, University of Naples Federico II, via Cintia 4, I-80126 Naples, Italy; \\ marialaura.alfieri@unina.it (M.L.A.); panzella@unina.it (L.P.); alesnapo@unina.it (A.N.); dischia@unina.it \\ (M.d.) \\ * Correspondence: rita.argenziano@unina.it; Tel.: +39-3312384925 \\ + Presented at the 1st International e-Conference on Antioxidants in Health and Disease, 1-15 December \\ 2020; Available online: https://cahd2020.sciforum.net/.
}

Published: 30 November 2020

\begin{abstract}
The development of innovative dip-coating technologies for surface functionalization has been a very active issue over the past decade following the discovery of the adhesion properties of polydopamine, a eumelanin-like material. New opportunities have derived from the discovery that hexamethylenediamine (HMDA) markedly enhances film deposition from a variety of catechol, including the key eumelanin precursor 5,6-dihydroxyindole (DHI). The remarkable antioxidant properties of synthetic eumelanins from the other main melanogenic precursor 5,6dihydroxyindole-2-carboxylic acid (DHICA) and its methyl ester (MeDHICA) have recently been described. In this work the film forming properties of MeDHICA melanin generated in the presence of HMDA or other diamines/monoamines and the antioxidant activity of the resulting films were investigated. Further to a systematic investigation, the most promising results were obtained running the aerobic oxidative polymerization of MeDHICA in aqueous buffers at $\mathrm{pH} 9.0$ at $1 \mathrm{mM}$ in the presence HMDA at 1:1.5 molar ratio. Under these conditions a dark yellow pigment is formed over $24 \mathrm{~h}$ exhibiting good film forming properties on different supports. HPLC analysis of the film solubilized in organic solvents indicated a mixture of oligomers of MeDHICA up to hexamers. Further polymerization of the film was obtained by exposure to ammonia vapors. The films showed high antioxidant activities in the 2,2-diphenyl-1-picrylhydrazyl and Ferric Reducing Antioxidant Power assays. Biocompatibility of MeDHICA/HMDA films was assessed on HaCat cells.
\end{abstract}

Keywords: antioxidant film; melanin; MeDHICA; PDA.

\section{Introduction}

The development of innovative and versatile dip-coating technologies for surface functionalization has been a very active issue over the past decade following the discovery of the extraordinary wet adhesion properties of polydopamine (PDA), a black insoluble and structurally disordered eumelanin-like material produced by the oxidative polymerization of dopamine under alkaline conditions. This material has been first described in 2007 following inspiration to the robust adhesion properties of mussel byssus proteins (Figure 1) [1]. These proteins, such as Mytilus edulis foot proteins-3 and -5 (Mfp-3 and -5), located in the distal portion of the mussel byssus where the byssal foot engages the substrate surface, have two key features that inspired PDA: 
The 1st International Electronic Conference on Antioxidants in Health and Disease, 1-15 December 2020

(1) high primary and secondary amine content due to lysine (Lys) and histidine (His) residues;

(2) high catechol content due to the presence of 3,4-dihydroxy-L-phenylalanine (DOPA).

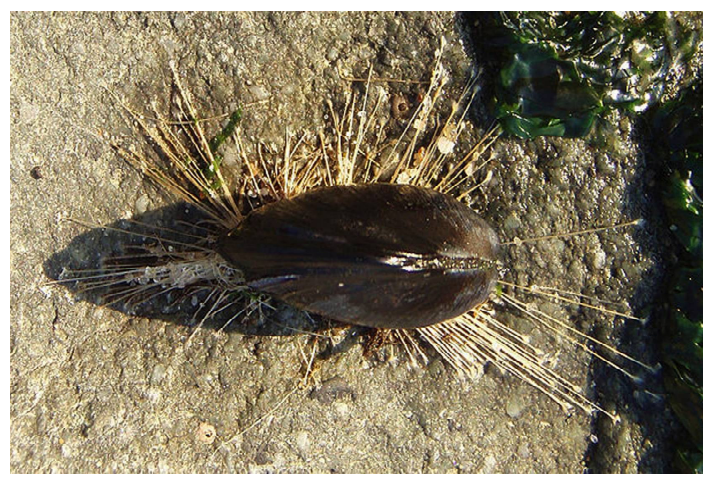

Figure 1. Mussel byssus.

An interplay of several chemical factors has been proposed to explain the excellent adhesion (between different materials) and cohesion (between different parts of the same material) properties of byssus proteins, such as H-bonding, electrostatic, hydrophobic, coordination and $\pi$-cation interactions [2]. The combination of the functional groups of the amino-acids lysine and DOPA of byssus proteins (Figure 2) allows PDA to form polymeric films that can be easily deposited at the interface of different materials and can be used to control or modify the hydrophobicity of a variety of interfaces [3].

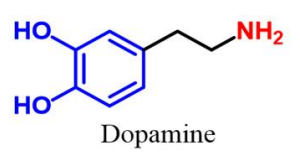

Figure 2. Dopamine structure highlighting the catechol (blue) and amine (red) components.

PDA film properties, including hydrophilicity and thickness, can be finely tuned by a variety of experimental parameters including dopamine concentration [4], nature of the buffer [5], oxidant [68], and pH. Polydopamine displays also many properties including optical, paramagnetic and electrical properties, and, most importantly, it exhibits excellent biocompatibility. Given these benefits, the use of polydopamine is not restricted to surface coating and has been rapidly extended to a wide range of applications across the chemical, biological, medical, and materials sciences, as well as in applied science engineering, and the technology fields [9]. In addition, in recent studies Chen et al. (2014) first reported that copolymerization of gallic acid and hexamethylenediamine (HMDA), a long chain aliphatic diamine, leads to films that can be deposited on various surfaces [10]. Addition of HMDA during PDA deposition was also proposed as a means of obtaining films rich in amine groups, with a high cross-linking degree and resistance to hydrolysis and swelling [11]. Moreover it was found that in the presence of HMDA the oxidation of caffeic acid, which itself has no adhesive properties despite apparent polymerization and darkening of the mixture, produces an adhesive greenish-blue material with high stability and biocompatibility [12]. Chemically stable functional biocompatible thin films were obtained also by a cross-linking reaction between pyrocatechol and HDMA under oxidizing condition[13].

New opportunities in PDA based surface chemistry have derived from the discovery that HMDA also induce film formation from DHI, which has been shown to give eumelanin-type polymers devoid of adhesion properties under PDA deposition conditions[14-16]. On this basis the research activity of the present work has been directed to assess whether HMDA can promote film deposition from other eumelanin precursors besides DHI, in particular 5,6-dihydroxyindole-2carboxylic acid (DHICA), the other main melanogenic precursor with high antioxidant activity and 
The 1st International Electronic Conference on Antioxidants in Health and Disease, 1-15 December 2020

to investigate the mechanisms by which HMDA can induce film deposition from non-adhesive melanin type polymers by defining structure-property relationships.

\section{Experiments Materials and Methods}

3,4-dihydroxy-L-phenylalanine (L-DOPA), potassium ferricyanide, sodium bicarbonate, sodium dithionite, hexamethylenediamine (HMDA), 1,2-ethylenediamine 1,4-butanediamine, 1,12dodecanediamine, butylamine, 2,2-diphenyl-1-picrilhydrazyl (DPPH), ferric chloride (III) hexahydrate and trispyridylhydrazine (TPTZ) were purchased by Sigma-Aldrich. The UV-vis spectra were recorded on a Jasco V-730 Spectrophotometer. HPLC analyses were performed on a Agilent 1100 binary pump instrument equipped with and a SPD-10AV VP UV-visible detector using an octadecylsilane-coated column, $250 \mathrm{~mm} \times 4.6 \mathrm{~mm}, 5 \mu \mathrm{m}$ particle size (Phenomenex Sphereclone ODS) at $0.7 \mathrm{~mL} / \mathrm{min}$ (detection wavelength was established at $300 \mathrm{~nm}$ ). Eluent system: Gradient of wateracetonitrile from $\mathrm{t} 5$ to $45 \mathrm{~min}$ from 40 to $70 \%$ of acetonitrile. Positive Reflectron MALDI spectra were recorded on a $\mathrm{AB}$ Sciex TOF/TOF 5800 instrument using 2,5-dihydroxybenzoic acid as the matrix. Substrates (quartzes, borosilicate coverslips) were cleaned by soaking in a solution of $\mathrm{H}_{2} \mathrm{SO}_{4} / 30 \%$ $\mathrm{H}_{2} \mathrm{O}_{2}$ 5:1 overnight, then cleaned with distilled water. Quartz substrates were dipped in the mixtures and after $24 \mathrm{~h}$ were rinsed with distilled water, sonicated in methanol/water solution 1:1 v/v, and airdried. The coated substrates thus obtained were analysed by ultraviolet-visible (UV-vis) spectrophotometry.

\subsection{Preparation of DHICA Methyl Ester (MeDHICA)}

DHICA methyl ester was obtained from DOPA methylester prepared by reacting DOPA (2.0 g) in methanol $(20 \mathrm{~mL})$ with $96 \%$ sulfuric acid $(2 \mathrm{~mL})$ under reflux. After $24 \mathrm{~h}$, the mixture was allowed to cool and sodium bicarbonate was added to neutrality. In addition, $1 \mathrm{~L}$ of bi-distilled water was degassed under argon flow. After $30 \mathrm{~min} 2 \mathrm{~g}$ of L-DOPA (final concentration $0.01 \mathrm{M}$ ), $13.4 \mathrm{~g}$ of potassium ferricyanide (4 molar equivalents) and $5 \mathrm{~g}$ of $\mathrm{NaHCO}_{3}$, dissolved in $50 \mathrm{~mL}$ of distilled water, were added. The mixture was kept under vigorous stirring and under argon flow for about 90 min, until the red color due to the dopacrome disappeared. The mixture was then treated with sodium dithionite, acidified to $\mathrm{pH} 3.0$ with $4 \mathrm{M} \mathrm{HCl}$ and extracted three times with ethyl acetate. The combined organic phases were dried with sodium sulfate and dried under vacuum to give a dark powder (1.3 g, 64\% yield) as a dark yellow-brown solid [17].

\subsection{Oxidative Polymerization of MeDHICA in the Presence of Amines}

A solution $1 \mathrm{mM}$ of MeDHICA at molar ratio 1:1 with different monoamines such as butylamine or diamines such as ethylenediamine, 1,4-butanediamine, hexamethylenediamine, 1,10diaminodecane and 1,12-dodecanediamine in $50 \mathrm{mM}$ carbonate buffer was stirred and a quartz substrate was dipped into the mixture. After $24 \mathrm{~h}$ the quartz was dried and analysed by UV-vis spectrophotometry. In control experiments the reaction was run in the absence of diamine or at different $\mathrm{pH}$ or under an argon atmosphere.

\subsection{DPPH, ABTS and FRAP Assay}

The assays were performed in this way: the glass substrates coated with MeDHICA/HMDA were immersed in an ethanolic solution of DPPH $50 \mu \mathrm{M}(20 \mathrm{~mL})$ or ABTS for $30 \mathrm{~min}$ and the antioxidant power was evaluated by UV-vis recording spectra every 5 min monitoring the progressive reduction of the absorbance at $515 \mathrm{~nm}$ for DPPH and at $734 \mathrm{~nm}$ for ABTS. In the case of the FRAP assay the development of a maximum at $594 \mathrm{~nm}$ was monitored [18-20]. 


\section{Results}

A first series of experiments were aimed at evaluating whether HMDA could promote film deposition from the eumelanin precursor MeDHICA, which can be prepared on gram scale following a procedure previously developed.The oxidative polymerization of MeDHICA was run in aqueous buffers at $\mathrm{pH} 9.0$ at different concentrations and in the presence of HMDA at different molar ratios. The most promising results were obtained using MeDHICA and HMDA at $1 \mathrm{mM}$ at 1:1.5 molar ratio. Under these conditions a light brown pigment is formed over $24 \mathrm{~h}$ exhibiting good film forming properties on quartz slides as shown in Figure 3.

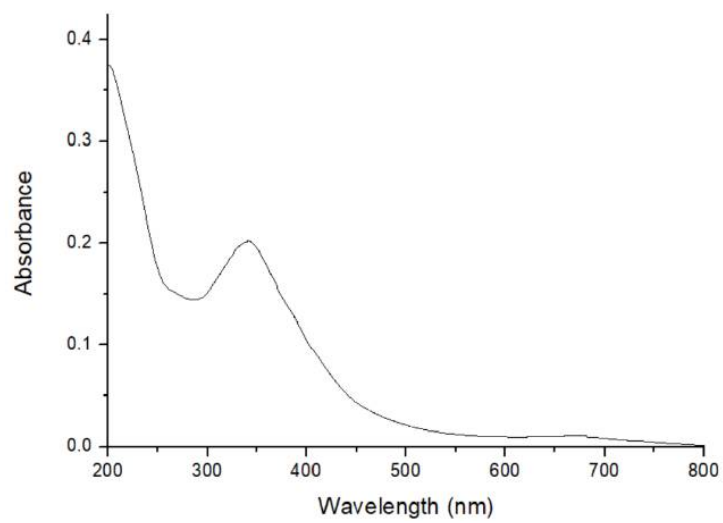

Figure 3. UV-vis spectrum of the quartz substrate dipped in the MeDHICA/HMDA mixture.

The film forming properties were observed on a variety of materials such as glass, polystyrene, polycarbonate (Figure 4)

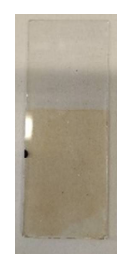

(a)

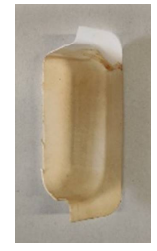

(b)

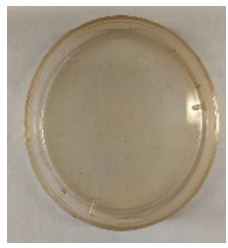

(c)

Figure 4. MeDHICA/HMDA coatings on (a) glass, (b) polyethylene, (c) polystyrene.

For comparison, the same reaction was carried out on MeDHICA at the same concentration but in the absence of HMDA, indicating that HMDA was critical to confer adhesive properties to MeDHICA. Various monoamines and diamines of different chain length were also investigated in place of HMDA, but no significant film deposition was observed in all cases. In a final set of experiments, the same reaction was run at different $\mathrm{pHs}$ and under argon atmosphere, but no film deposition was observed under these latter conditions.To clarify the mechanism underlying film deposition, the quartz surface of MeDHICA/HMDA was dissolved in methanol and the resulting solution was subjected to HPLC (Figure 5) and MALDI analysis. 
The 1st International Electronic Conference on Antioxidants in Health and Disease, 1-15 December 2020

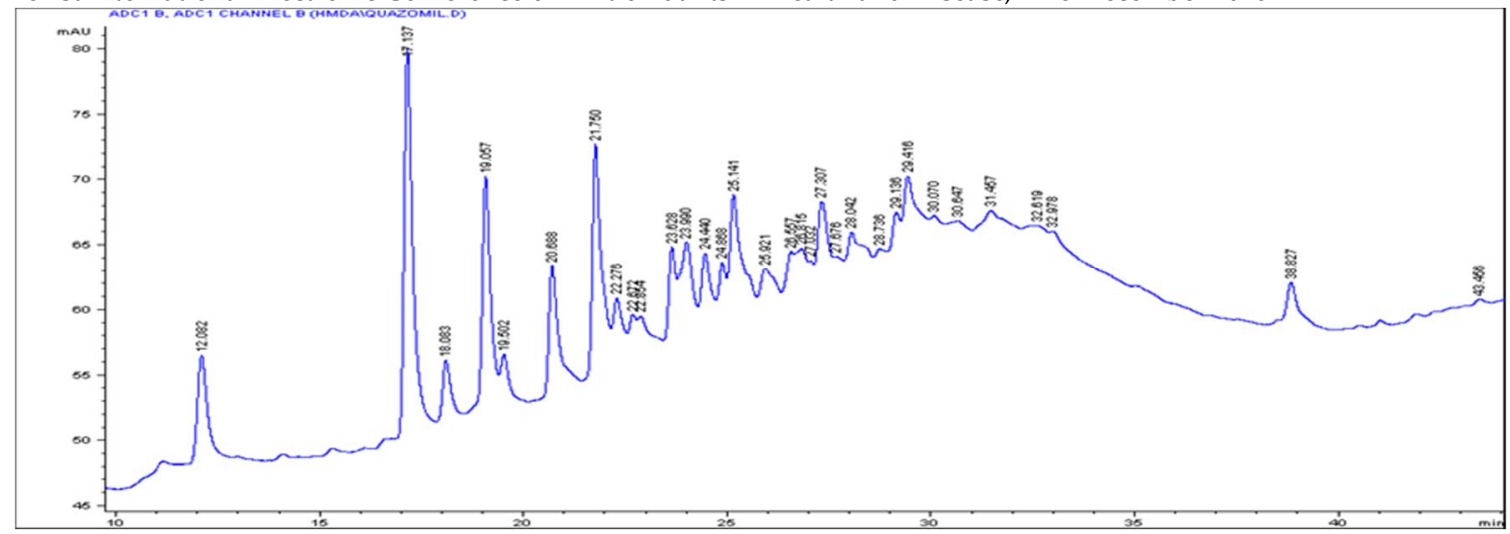

Figure 5. Elutographic profile of the material deposited on the quartz slide dissolved in DMSO. UV detection at $300 \mathrm{~nm}$.

The chromatographic profile showed the presence of MeDHICA (Rt $12 \mathrm{~min}$ ), a dimer (Rt $17 \mathrm{~min}$ ) and other oligomeric species, suggesting that the coating is composed of a mixture of low oligomeric species. This was also confirmed by MALDI analysis (Figure 6).
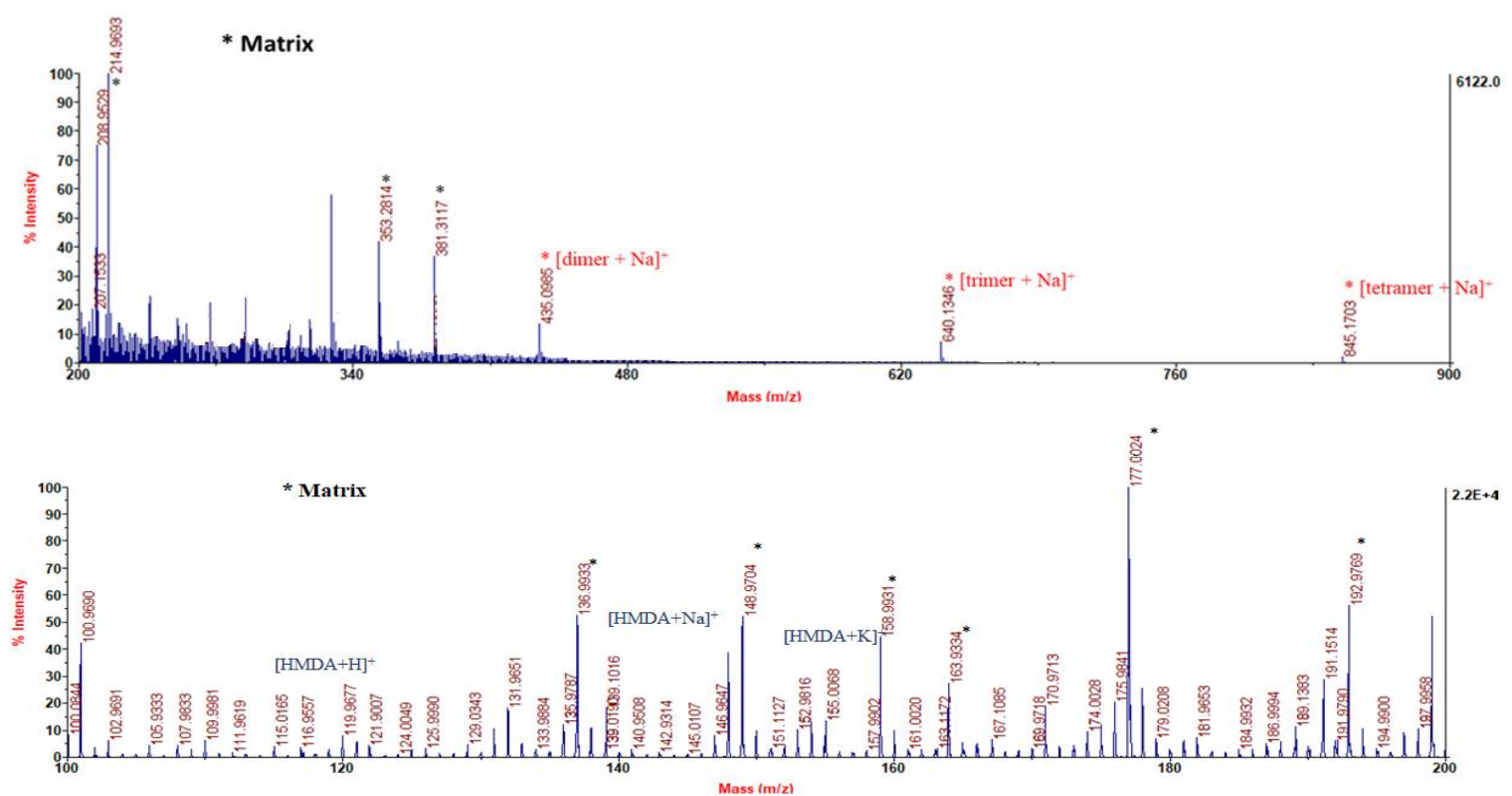

Figure 6. Mass Spectrum MALDI of the film that was directly deposited on the MALDI plate.

The antioxidant properties of the MeDHICA/HMDA film were then evaluated by the 2,2diphenyl-1-picrilhydrazyl-(DPPH) assay. To this aim, glass substrates obtained as described above were immersed in a beaker containing $20 \mathrm{~mL}$ of a solution of DPPH $(50 \mu \mathrm{M})$ in methanol care being taken to dip all the glass slides up to a fixed level into the solution. The absorption of the DPPH solution was monitored over time. The same procedure was followed for other antioxidant assays, i.e the 2,2'-azino-bis-(3-ethylbenzothiazoline-6-sulfonic acid)-ABTS and Ferric reducing antioxidant power- FRAP (Figure 7). 


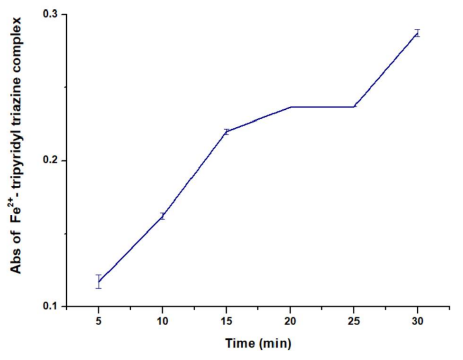

(a)

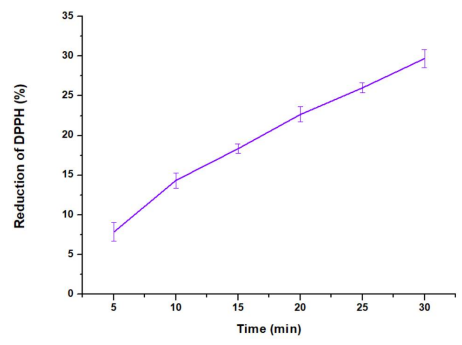

(b)

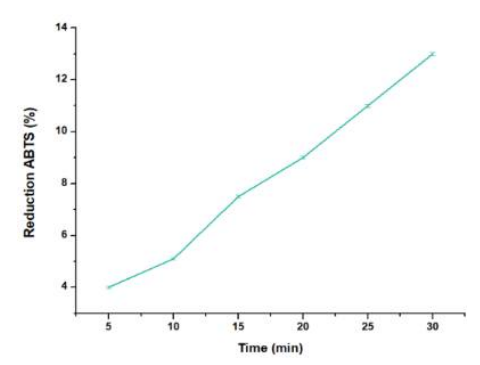

(c)

Figure 7. (a) Kinetics of development of the absorption of $\mathrm{Fe}^{2+}$-tripyridyl triazine complex (b) reduction of DPPH $(50 \mu \mathrm{M})$ and $(\mathbf{c})$ ABTS $(50 \mu \mathrm{M})$ over time by immersion of glass substrates MeDHICA/HMDA.

MeDHICA/HMDA film obtained after $6 \mathrm{~h}$ showed a $50 \%$ higher antioxidant power with respect to those obtained after $24 \mathrm{~h}$ of dipping (Figure 8).

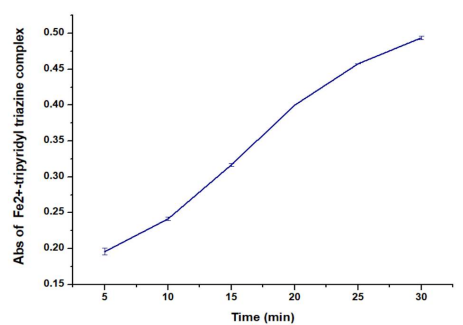

(a)

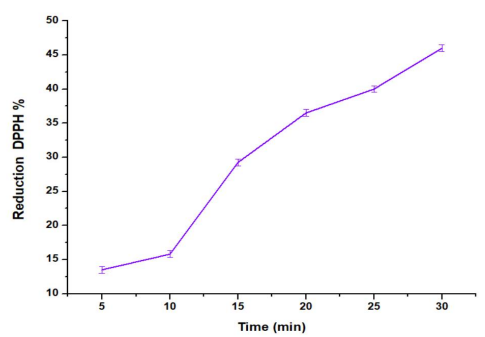

(b)

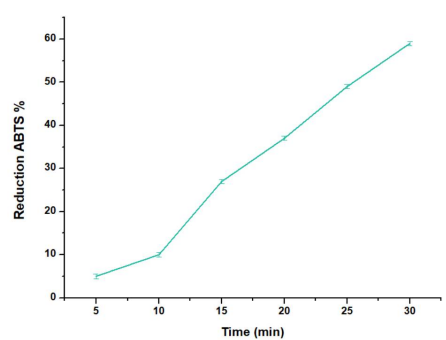

(c)

Figure 8. (a) Kinetics of development of the absorption of $\mathrm{Fe}^{2+}$-tripyridyl triazine complex (b) reduction of DPPH $(50 \mu \mathrm{M})$ and $(\mathbf{c})$ ABTS $(50 \mu \mathrm{M})$ over time by immersion of glass substrates MeDHICA/HMDA.

\section{Discussion}

The systematic investigation of the aerobic oxidation of MeDHICA in aqueous buffers at $\mathrm{pH} 9.0$ at different concentrations and in the presence of various diamines and monoamines at different molar ratios indicated as the best conditions the reaction run using MeDHICA and HMDA at $1 \mathrm{mM}$ at 1:1.5 molar ratio. Good film forming properties on various materials were observed for the yellow dark pigment formed under these conditions. In addition this film was endowed of high antioxidant activity. The chemical composition of the film as investigated by HPLC and MALDI analysis indicated a mixture of low oligomers of the indole and the presence of HMDA not linked to the oligomer omponets. On this basis, it is proposed an adhesion mechanism, based on $\pi$-cations interactions. (Figure 9).
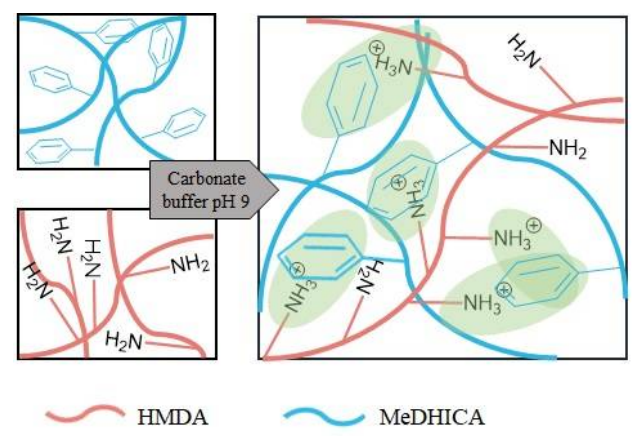
Figure 9. Adhesion mechanism of the film MeDHICA/HMDA.

Further investigation will be devoted at evaluating the applications of the films from MeDHICA/HMDA e.g., in wound healing or tissue repair starting from in vitro cellular models.

\section{Conclusions}

Based on the results of the present work it is concluded that the combination of a long aliphatic chain with two amine groups is crucial to confer to catechol polymers a suitable balance of hydrophobicity, aggregation, and crosslinking. In addition based on HPLC and MALDI analysis is proposed a mechanism based on an interaction between the aromatic ring of MeDHICA and also its oligomeric species with the amine groups of HMDA, which is protonated under the reaction conditions.

Author Contributions: A.N. conceived and designed the experiments; R.A. and M.L.A. performed the experiments; L.P. analyzed the data; M.d. wrote the paper. All authors have read and agreed to the published version of the manuscript.

Funding: This research received no external funding.

Acknowledgments: This work was supported in part by Italian MIUR grants PRIN 2017YJMPZN to MdI.

Conflicts of Interest: The authors declare no conflict of interest.

\section{Abbreviations}

The following abbreviations are used in this manuscript:

$\begin{array}{ll}\text { HMDA } & \text { Hexamethylenediamine } \\ \text { DHI } & \text { 5,6-dihydroxyindole } \\ \text { DHICA } & \text { 5,6-dihydroxyindole-2-carboxylic acid } \\ \text { MeDHICA } & \text { Methyl ester 5,6-dihydroxyindole-2-carboxylic acid } \\ \text { HPLC } & \text { High performance liquid chromatography } \\ \text { MALDI } & \text { Matrix assisted laser desorption ionisation } \\ \text { DPPH } & \text { 2,2-diphenyl-1-picrilhydrazyl radical } \\ \text { ABTS } & \text { 2,2'-azino-bis-(3-ethylbenzothiazoline-6-sulfonic acid) } \\ \text { FRAP } & \text { Ferric reducing antioxidant power }\end{array}$

\section{References}

1. Ryu, J.H.; Messersmith, P.B.; Lee, H. Polydopamine Surface Chemistry: A Decade of Discovery. ACS Appl. Mater. Interfaces 2018, 10, 7523-7540, doi:10.1021/acsami.7b19865.

2. Waite, J.H. Mussel adhesion-Essential footwork. J. Exp. Biol. 2017, 220, 517-530, doi:10.1242/jeb.134056.

3. Lee, B.P.; Messersmith, P.B.; Israelachvili, J.N.; Waite, J.H. Mussel-Inspired Adhesives and Coatings. Annu. Rev. Mater. Res. 2011, 41, 99-132, doi:10.1146/annurev-matsci-062910-100429.

4. Ball, V.; Del Frari, D.; Toniazzo, V.; Runch, D. Kinetics of polydopamine film deposition as a function of $\mathrm{pH}$ and dopamine concentration: Insights in the polydopamine deposition mechanism J. Colloid Interface Sci. 2012, 386, 366-372, doi:10.1016/j.jcis.2012.07.030.

5. Bernsmann, F.; Ball, V.; Addiego, F.; Ponche, A.; Michel, M.; Gracio, J.J.D.A.; Toniazzo, V.; Ruch, D. Dopamine-melanin film deposition depends on the used oxidant and buffer solution. Langmuir 2011, 27, 2819-2825, doi:10.1021/la104981s.

6. Wei, Q.; Zhang, F.; Li, J.; Li, B.; Zhao, C. Oxidant-induced dopamine polymerization for multifunctional coatings. Polym. Chem. 2010, 1, 1430-1433, doi:10.1039/C0PY00215A.

7. Du, X.; Li, L.; Li, J.; Yang, C.; Frenkel, N.; Welle, A.; Heissler, S.; Nefedov, A.; Grunze, M.; Levkin, P.A. UVTriggered Dopamine Polymerization: Control of Polymerization, Surface Coating, and Photopatterning Adv. Mater. 2014, 26, 8029-8033, doi:10.1002/adma.20140370. 
The 1st International Electronic Conference on Antioxidants in Health and Disease, 1-15 December 2020

8. Ponzio, F.; Barthès, J.; Bour, J.; Michel, M.; Bertani, P.; Hemmerlé, J.; d'Ischia, M.; Ball, V. Oxidant Control of Polydopamine Surface Chemistry in Acids: A Mechanism-Based Entry to SuperhydrophilicSuperoleophobic Coatings. Chem. Mater. 2016, 28, 4697-4705, doi:10.1021/acs.chemmater.6b01587.

9. Liu, Y.; Ai, K.; Lu, L. Polydopamine and its derivative materials: Synthesis and promising applications in energy, environmental, and biomedical fields. Chem. Rev. 2014, 114, 5057-5115, doi:10.1021/cr400407a.

10. Chen, S.; Li, X.; Yang, Z.; Zhou, S.; Luo, R.; Maitz, M.F.; Zhao, Y.; Wang, J.; Xiong, K.; Huang, N. A simple one-step modification of various materials for introducing effective multi-functional groups. Colloids Surf. B Biointerfaces 2014, 113, 125-133, doi:10.1016/j.colsurfb.2013.08.041.

11. Yang, Y.; Qi, P.; Ding, Y.; Maitz, M.F.; Yang, Z.; Tu, Q.; Xiong, K.; Leng, Y.; Huang, N. A biocompatible and functional adhesive amine-rich coating based on dopamine polymerization. J. Mater. Chem. B 2015, 3, 7281, doi:10.1039/C4TB01236D.

12. Iacomino, M.; Paez, J.I.; Avolio, R.; Carpentieri, A.; Panzella, L.; Falco, G.; Pizzo, E.; Errico, M.E.; Napolitano, A.; del Campo, A. Multifunctional Thin Films and Coatings from Caffeic Acid and a CrossLinking Diamine. Langmuir 2017, 33, 2096-2102, doi:10.1021/acs.langmuir.6b04079.

13. Suárez-García, S.; Sedó, J.; Saiz-Poseu, J.; Ruiz-Molina, D. Copolymerization of a Catechol and a Diamine as a Versatile Polydopamine-Like Platform for Surface Functionalization: The Case of a Hydrophobic Coating Biomimetics 2017, 2, 22, doi:10.3390/biomimetics2040022.

14. Alfieri, M.L.; Panzella, L.; Oscurato, S.L.; Salvatore, M.; Avolio, R.; Errico, M.E.; Maddalena, P.; Napolitano, A.; d'Ischia, M. The Chemistry of Polydopamine Film Formation: The Amine-Quinone Interplay Biomimetics 2018, 3, 26, doi:10.3390/biomimetics3030026.

15. Alfieri, M.L.; Micillo, R.; Panzella, L.; Crescenzi, O.; Oscurato, S.L.; Maddalena, P.; Napolitano, A.; Ball, V.; d'Ischia, M. Structural Basis of Polydopamine Film Formation: Probing 5,6-Dihydroxyindole-Based Eumelanin Type Units and the Porphyrin Issue ACS Appl. Mater. Interfaces 2018, 10, 7670-7680, doi.org/10.1021/acsami.7b09662.

16. Alfieri, M.L.; Panzella, L.; Oscurato, S.L.; Salvatore, M.; Avolio, R.; Errico, M.E.; Maddalena, P.; Napolitano, A.; Ball, V.; d'Ischia, M. A Clean and Tunable Mussel-Inspired Coating Technology by Enzymatic Deposition of Pseudo-Polydopamine ( $\psi$-PDA) Thin Films from Tyramine. Front. Chem. 2019, 7, 407, doi:10.3390/ijms21144873.

17. Edge, R.; d'Ischia, M.; Land, E.J.; Napolitano, A.; Navaratnam, S.; Panzella, L.; Pezzella, A.; Ramsden, A.C.; Riley, P.A. Dopaquinone redox exchange with dihydroxyindole and dihydroxyindole carboxylic acid Pigment Cell Res. 2006, 19, 443-450, doi:10.1111/j.1600-0749.2006.00327.

18. Goupy, P.; Dufour, C.; Loonis, M.; Dangles, O. Quantitative kinetic analysis of hydrogen transfer reactions from dietary polyphenols to the DPPH radical. J. Agric. Food Chem. 2003, 51, 615-622, doi:10.1021/jf0259381.

19. Benzie, I.F.F.; Strain, J.J. The ferric reducing ability of plasma (FRAP) as a measure of "antioxidant power": The FRAP assay. Anal. Biochem. 1996, 239, 70-76, doi:10.1006/abio.1996.0292.

20. Re, R.; Pellegrini, N.; Proteggente, A.; Pannala, A.; Yang, M.; Rice-Evans, C. Antioxidant activity applying an improved ABTS radical cation decolorization assay. Free Radic. Biol. Med. 1999, 26, 1231-1237, doi:10.1016/S0891-5849(98)00315-3.

(c) 2020 by the authors; licensee MDPI, Basel, Switzerland. This article is an open access article distributed under the terms and conditions of the Creative Commons by Attribution (CC-BY) license (http://creativecommons.org/licenses/by/4.0/). 